九州大学学術情報リポジトリ

Kyushu University Institutional Repository

\title{
Study on Characteristics of Paper laminated with Biodegradable Plastics (3) : Elemental and Morphological Analyses of Polylactide Deterioration
}

\section{Mayumi, Ayaka}

Laboratory of Bioresources Chemistry, Division of Biomaterial Science, Department of Forest and Forest Products Sciences, Graduate School of Bioresource and Bioenvironmental Sciences, Kyushu University

\section{Kanie, Osamu}

Laboratory of Bioresources Chemistry, Division of Biomaterial Science, Department of Forest and Forest Products Sciences, Graduate School of Bioresource and Bioenvironmental Sciences, Kyushu University

\section{Kitaoka, Takuya}

Laboratory of Bioresources Chemistry, Division of Biomaterial Science, Department of Forest and Forest Products Sciences, Graduate School of Bioresource and Bioenvironmental Sciences, Kyushu University

Wari ishi, Hiroyuki

他

https://doi.org/10.5109/4531

出版情報: 九州大学大学院農学研究院紀要. 48 (1/2)，pp.97-106，2003-10-01. Faculty of Agriculture, Kyushu University

バージョン :

権利関係 : 


\title{
Study on Characteristics of Paper Laminated with Biodegradable Plastics, 3. Elemental and Morphological Analyses of Polylactide Deterioration
}

\author{
Ayaka MAYUMI*, Osamu KANIE*, Takuya KITAOKA', \\ Hiroyuki WARIISHI and Hiroo TANAKA
}

\author{
Laboratory of Bioresources Chemistry, Division of Biomaterial Science, Department of \\ Forest and Forest Products Sciences, Faculty of Agriculture, \\ Kyushu University, Fukuoka 812-8581, Japan \\ (Received March 10, 2003 and accepted July 15, 2003)
}

\begin{abstract}
Elemental and morphological analyses were applied to investigate the degradation behavior of commercial polylactide (PLA) film, poly (L-lactide) (PLLA) and poly (DL-lactide) (PDLLA) by $\mathrm{X}$-ray photoelectron spectroscopy (XPS) and atomic force microscopy (AFM), respectively. The PLLA and PDLLA samples consisted of three types of carbon: $\mathrm{C}-\mathrm{C}(\mathrm{C}-\mathrm{H}), \mathrm{C}-\mathrm{O}$ and $\mathrm{O}=\mathrm{C}-\mathrm{O}$, which corresponded to their theoretical composition. However, the XPS analysis revealed that the surfaces of the PLA film highly resistant to acid hydrolysis were overspread with unknown constituents consisting of nitrogen and unoxidized carbon, not being announced officially. The AFM analysis offered a clear distinction in the surface deterioration of the PLA samples; and the alkaline treatment excavated numerous holes on the PLA surfaces. These results indicated that the commercial plastics are chemically modified on their surfaces to improve the physical properties for practical use, resulting in being deprived of biodegradability and eco-compatibility. It was also suggested that the enlargement in the specific surface area, which may occur at the early stage on PLA degradation, possibly accelerated the PLA decomposition rate. A new conception in the material design for paper-based composites was proposed; a local alkaline circumstance expected by alkaline fillers, e.g., $\mathrm{CaO}$ available in papermaking processes would effectively promote the PLA decomposition.
\end{abstract}

\section{INTRODUCTION}

Ester-type biodegradable plastics, which are represented by the following; polylactide (PLA), poly ( $\beta$-hydroxybutyrate), poly ( $\varepsilon$-caprolactone) and their copolymers, have a great possibility for resolving the material circulation and the waste concern on forthcoming biomass-based society (Ikada et al., 2000; Mohanty et al., 2000). However, they have a recent tendency to be overestimated: most of them commercially available seem not to degrade in natural environments (Kanie et al., 2002). In general, it is sure that polyester is easily hydrolyzed under both acidic and alkaline conditions. However, the PLA products used in our previous study were allowed to accept only alkaline hydrolysis (Mayumi et al., 2003), while the alkaline hydrolysis cannot generally occur in natural environments under the acidic-neutral conditions. Therefore, a further progress in the researches for material biodegradation is required to elucidate the decomposition

\footnotetext{
* Laboratory of Bioresources Chemistry, Division of Biomaterial Science, Department of Forest and Forest Products Sciences, Graduate School of Bioresource and Bioenvironmental Sciences, Kyushu University, Fukuoka 812-8581, Japan

+ Corresponding author (E-mail: tkitaoka@agr.kyushu-u.ac.jp)
} 
behavior and mechanism, which would be applied to a material design with high eco-compatibility.

Degradation mechanism of polyesters in water and soil was proposed as follows: (1) non-enzymatic hydrolysis of the polyesters occurs at first, followed by the conversion to low molecular weight compounds, and (2) they are metabolized to $\mathrm{CO}_{2}$ and $\mathrm{H}_{2} \mathrm{O}$ by various in-situ microorganisms (Tsuji et al., 2001). In other words, at the first step an abiotic attack occurs on the solid surfaces of polymeric materials, and then a sequential biotic assault immediately takes place. However, the PLA plastics received nearly no damage in soil as described above. Therefore, the delicate phenomena at the interface on the PLA solid are of very significance for evaluating the material degradation. In particular, elemental and morphological characteristics on the degradation of polymeric products should be one of the most striking factors, and must be investigated in detail for the material biodegradability.

In this study, surface properties of various PLA products and characteristic changes in the surface degradation were investigated by X-ray photoelectron spectroscopy (XPS) and atomic force microscopy (AFM). Chemical and biological deterioration of PLAs were characterized from the results obtained through the surface analyses, and a new conception for an ecomaterial design that the PLA having nearly no alkaline resistance is compounded with the paperboard containing alkaline fillers as functional additives was proposed on the basis of the findings obtained.

\section{MATERIALS AND METHODS}

\section{Materials}

PLA film (Ecoloju; 25 $\mu \mathrm{m}$; biaxially oriented; Mitsubishi Plastics Co. Ltd., Japan) and its laminated paperboard were commercial products. Poly (L-lactide) (PLLA) and poly (DL-lactide) (PDLLA) were provided by Birmingham Polymers Co. Ltd., USA. Commercial polyethyleneterephtalate (PET; $23 \mu \mathrm{m}$; biaxially oriented) film was purchased from Goodfellow Cambridge Co. Ltd., UK. Other chemicals were reagent grade (Wako Pure Chemical Industries Co. Ltd., Japan) and used without further purification.

\section{Procedures}

Chemical treatment was carried out using $1 \mathrm{M}$ hydrochloric acid or $1 \mathrm{M}$ sodium hydroxide at room temperature for a designated time: 0,1 or 4 hours. Biological stress was provided by the burial test in soil for $0,1,6,12$ or 24 months, as referred in part to our previous study (Kanie et al., 2002). Sample specimens were thoroughly washed with deionized water, and then air-dried without any thermal treatment before the surface analyses in the following section. For a model treatment with alkaline fillers, PLLA and PDLLA beads $(0.1 \mathrm{~g}$ ) was suspended in $25 \mathrm{ml}$ of $2 \%$ (wt/vol) filler suspension containing $\mathrm{CaCO}_{3}, \mathrm{CaO}, \mathrm{MgCO}_{3}$ or $\mathrm{MgO}$ at $20^{\circ}$ or $80^{\circ} \mathrm{C}$.

\section{Analyses}

Elemental analysis of material surfaces was performed using an AXIS-HSi spectrometer (Shimadzu/Kratos Co. Ltd., Japan) equipped with a monochromatic AlK $\alpha \mathrm{X}$-ray 
source $(1486.6 \mathrm{eV})$ under the $\mathrm{X}$-ray generation conditions at a voltage of $15 \mathrm{kV}$ and a current of $10 \mathrm{~mA}$ below $5 \times 10^{-7} \mathrm{~Pa}$ (Yamamoto et al., 2003). Pass energy and step width were set at $10 \mathrm{eV}$ and $0.05 \mathrm{eV}$, respectively. Binding energies of all spectra were related to a conspicuous $\mathrm{C} 1 \mathrm{~s}$ signal (unoxidized $\mathrm{C}-\mathrm{C}$ ) at $285 \mathrm{eV}$. Morphological analysis of the PLA surfaces before and after exposure to chemical or biological stress was carried out by tapping-mode AFM using a NanoScope IIIa microscope (Digital Instruments Co. Ltd., USA) with a silicon-single-crystal microcantilever probe under ambient conditions. The probe tip radius and spring constant were in the ranges of 5-10 nm and $20-100 \mathrm{~N} / \mathrm{m}$, respectively, as announced supplier's values. Topographic images were obtained with a resonance frequency of approximately $200-400 \mathrm{kHz}$ for the probe oscillation. Scanning rate of piezometer was $0.3 \mathrm{~Hz}$, and the pictures were horizontally image-captured at $2^{8} \times 2^{8}$ pixels. Roughness of the sample surface was evaluated by a root mean square (RMS) value.

\section{RESULTS AND DISCUSSION}

\section{XPS and AFM analyses of PLA surfaces on chemical treatments}

Figures 1 and 2 illustrate the XPS survey- and narrow-scanned spectra of PLA samples, respectively. PLA originally consists of carbon, oxygen and hydrogen (Fig. 1a). However, the nitrogen components were detected on the commercial PLA film (Fig. 1b),

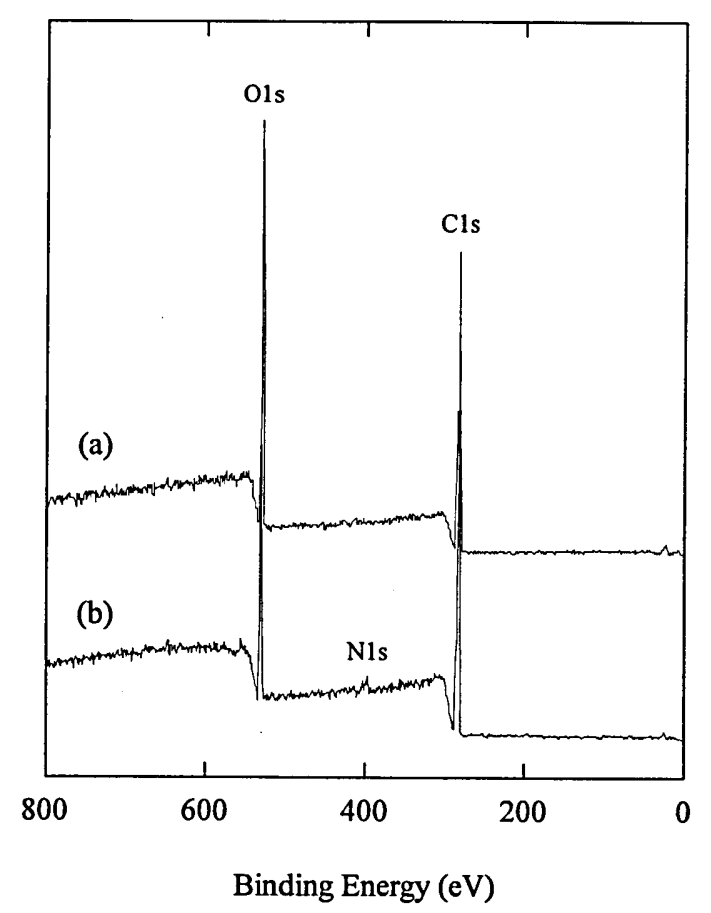

Fig. 1. XPS survey spectra of pure PLA sample (a) and commercial PLA film (b). 


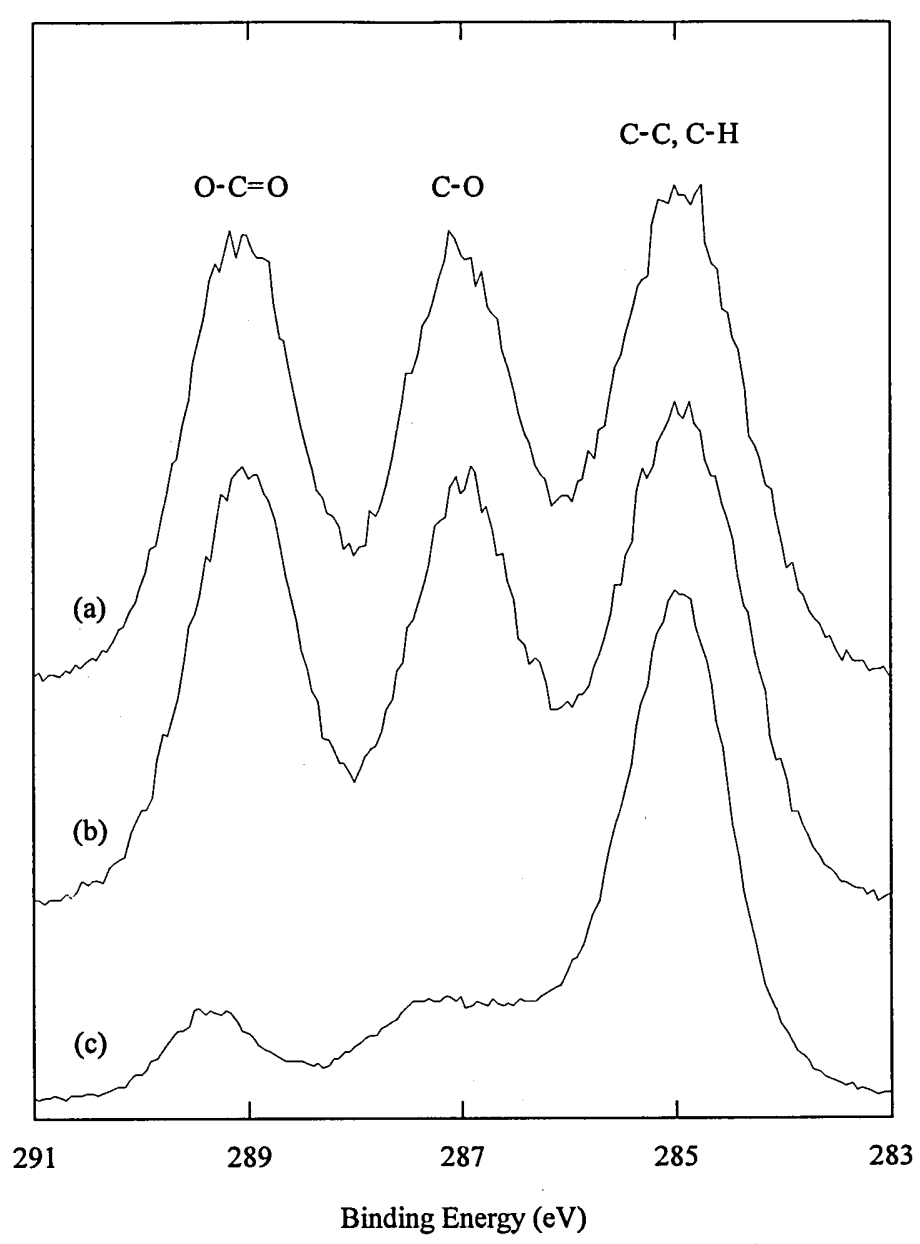

Fig. 2. XPS C1s narrow spectra of PLLA (a), PDLLA (b) and commercial PLA film (c).

in accord with the previous result for the characteristic Raman band from $\mathrm{N}-\mathrm{H}$ stretching (Mayumi et al., 2003). Moreover, the atomic ratio of carbon: $\mathrm{C}-\mathrm{C}(\mathrm{C}-\mathrm{H}), \mathrm{C}-\mathrm{O}$ and $\mathrm{O}=\mathrm{C}-\mathrm{O}$ corresponded well to the theoretical and stoichiometric value of 1:1:1 in the PLLA and PDLLA samples as shown in Figs. 2a and 2b; however a remarkable strong peak of $\mathrm{C}-\mathrm{C}$ $(\mathrm{C}-\mathrm{H})$ at $285 \mathrm{eV}$, being about seven times as large as those of $\mathrm{C}-\mathrm{O}$ and $\mathrm{O}=\mathrm{C}-\mathrm{O}$, was observed in the commercial PLA film surfaces, as shown in Fig. 2c. These XPS results implied the surface modification for improving the physical properties of PLA products in exchange for the biodegradability, not being announced officially.

Figure 3 visualizes the AFM images of the surfaces of PLA and PET films treated by $1 \mathrm{M} \mathrm{HCl}$ for 0,1 or 4 hours. Table 1 lists the RMS values obtained from the images in Fig. 

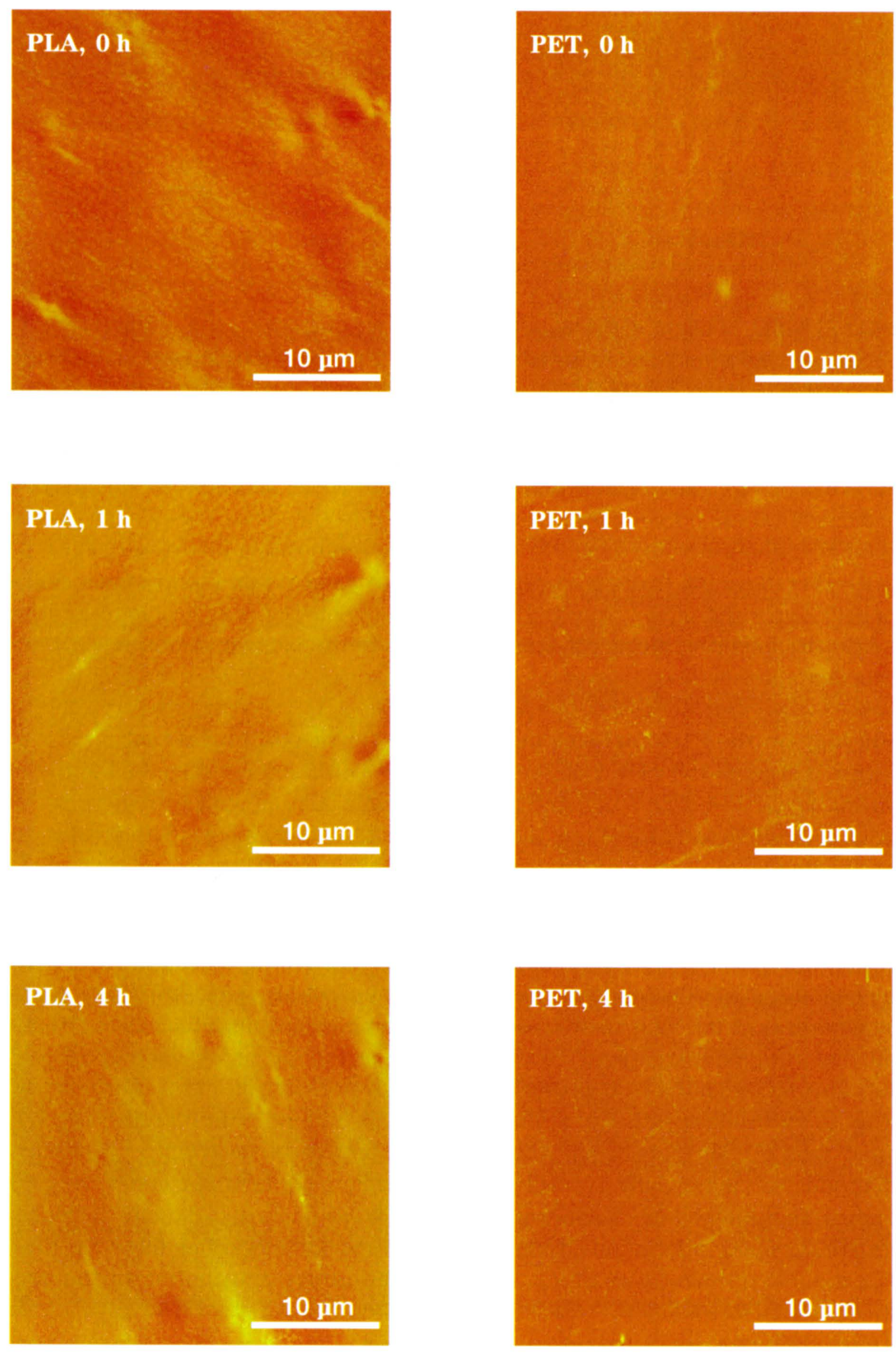

Fig. 3. AFM images of the surfaces of PLA and PET films by chemical treatment with $1 \mathrm{M} \mathrm{HCl}$ at $20^{\circ} \mathrm{C}$. 
Table 1. Root mean square (RMS) values of PLA and PET films by chemical treatments.

\begin{tabular}{lccc}
\hline & & \multicolumn{2}{c}{ RMS (nm \pm SD) } \\
\cline { 3 - 4 } & & PLA film & PET film \\
\hline \multirow{2}{*}{ Untreated } & & $5.75 \pm 0.42$ & $0.61 \pm 0.08$ \\
\hline \multirow{2}{*}{$1 \mathrm{M} \mathrm{HCl}$} & $1 \mathrm{~h}$ & $5.98 \pm 0.47$ & $0.85 \pm 0.19$ \\
& $4 \mathrm{~h}$ & $7.32 \pm 2.73$ & $0.98 \pm 0.46$ \\
\hline \multirow{2}{*}{$1 \mathrm{M} \mathrm{NaOH}$} & $1 \mathrm{~h}$ & $25.7 \pm 9.92$ & $0.77 \pm 0.19$ \\
& $4 \mathrm{~h}$ & $61.2 \pm 11.9$ & $1.00 \pm 0.27$ \\
\hline
\end{tabular}

3. In the PLA film, a slight disturbance on the surfaces was observed, while the PET surfaces remained flat after the 4-hour $\mathrm{HCl}$ treatment. The acidic treatment made nearly no influence on the surfaces of both film samples, and resulted in the almost constant RMS values in Table 1. Figure 4 shows a clear difference in the surface morphology by the $\mathrm{NaOH}-$ treatment, increasing excavated holes only on the PLA surfaces as the treatment time proceeded. In contrast, the PET surfaces were never damaged by the alkali. The morphological variation approximately corresponded to the surface roughening behavior; and the RMS values increased rapidly as the PLA decomposition progressed, as shown in Table 1. Therefore, the hydrolysis of PLA products occurred on the interface, and then proceeded nearby the region damaged initially. The heterogeneous decomposition would accelerate the following biological accessibility when the biodegradation might occur in natural environments.

\section{Morphology on the PLA surfaces before and after in-soil burial test}

Figure 5 displays the AFM images of the surfaces of PLA films subjected to the burial test in soil for $0,1,6,12$ or 24 months. No obvious variation appeared, however slight marks of erosion were observed as the burial periods went longer. The erosion traces were estimated at sub-micron holes in diameter. These samples were not degraded by a judgment based on the visual inspection (Kanie et al., 2002); however, the slightest biodegradation was confirmed on the interface, not being reflected in the loss in sample weight and the other analytical results (Mayumi et al., 2003). It was obscure whether these phenomena were induced by non-enzymatic hydrolysis or by direct biotic attacks. The comparison of the PLA degradation under the chemical and biological stress suggested that the alkali treatment must be effective for the quick decomposition and possibly for promoting the biodegradation by in-situ microorganisms. Unfortunately, the $\mathrm{pH}$ of water and soil recently has a tendency to shift from neutral to acidic, e.g., by acid rain, and thus the alkaline circumstances cannot be expected actually. However, the paper products commercially available are practically manufactured under the alkaline conditions since a plenty of calcium carbonate is used as fillers and the $\mathrm{CaCO}_{3}$-containing papers are recycled in large quantities. Thus, the effects of various fillers on the PLA deterioration were investigated for a new material design contributing to the actually effective material degradation.

\section{Possibility of alkaline fillers as degradation enhancer}

Table 2 represents the $\mathrm{pH}$ of $2 \%$ (wt/vol) filler suspension containing $\mathrm{CaCO}_{3}, \mathrm{CaO}$, 

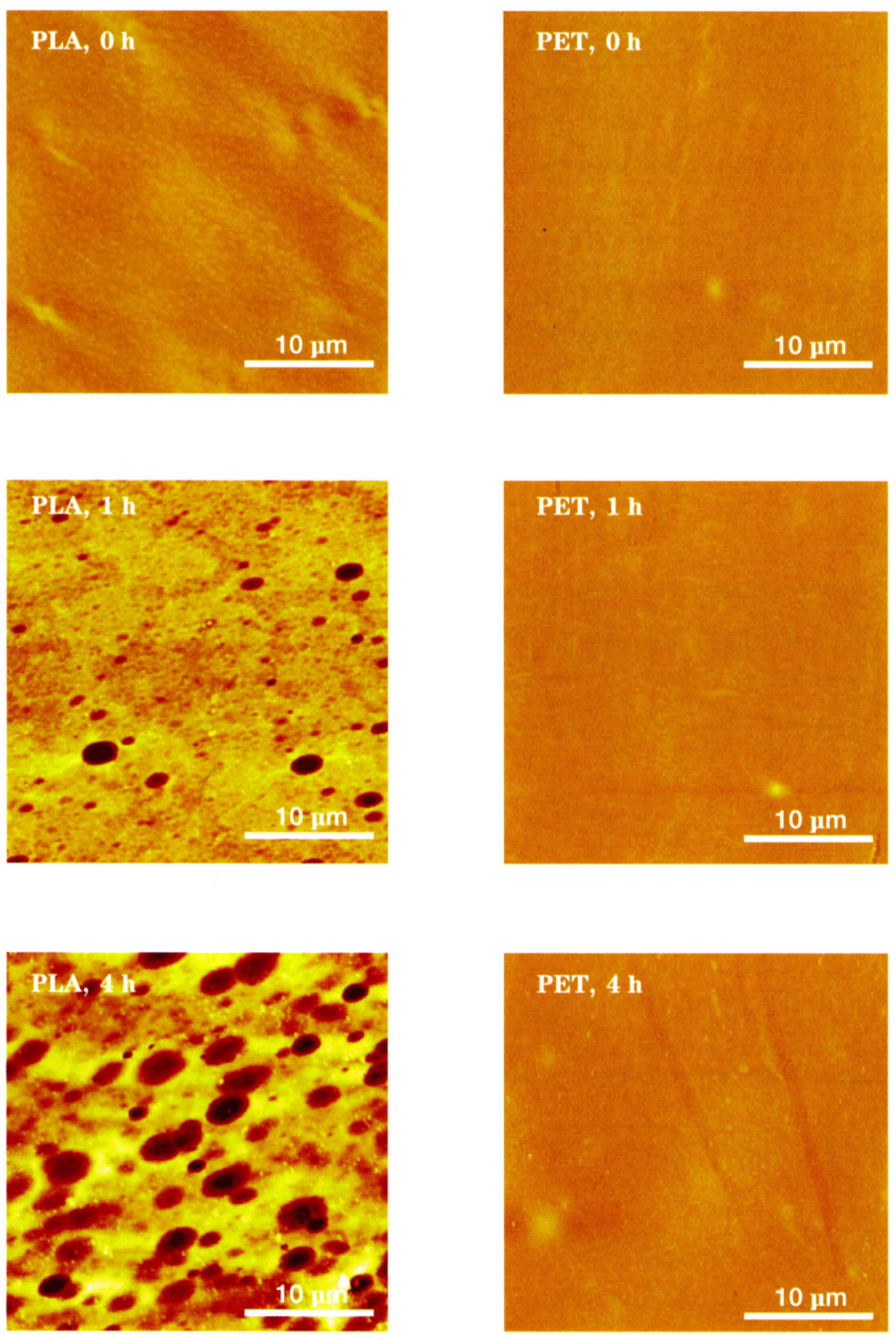

Fig. 4. AFM images of the surfaces of PLA and PET films by chemical treatment with $1 \mathrm{M}$ $\mathrm{NaOH}$ at $20^{\circ} \mathrm{C}$. 

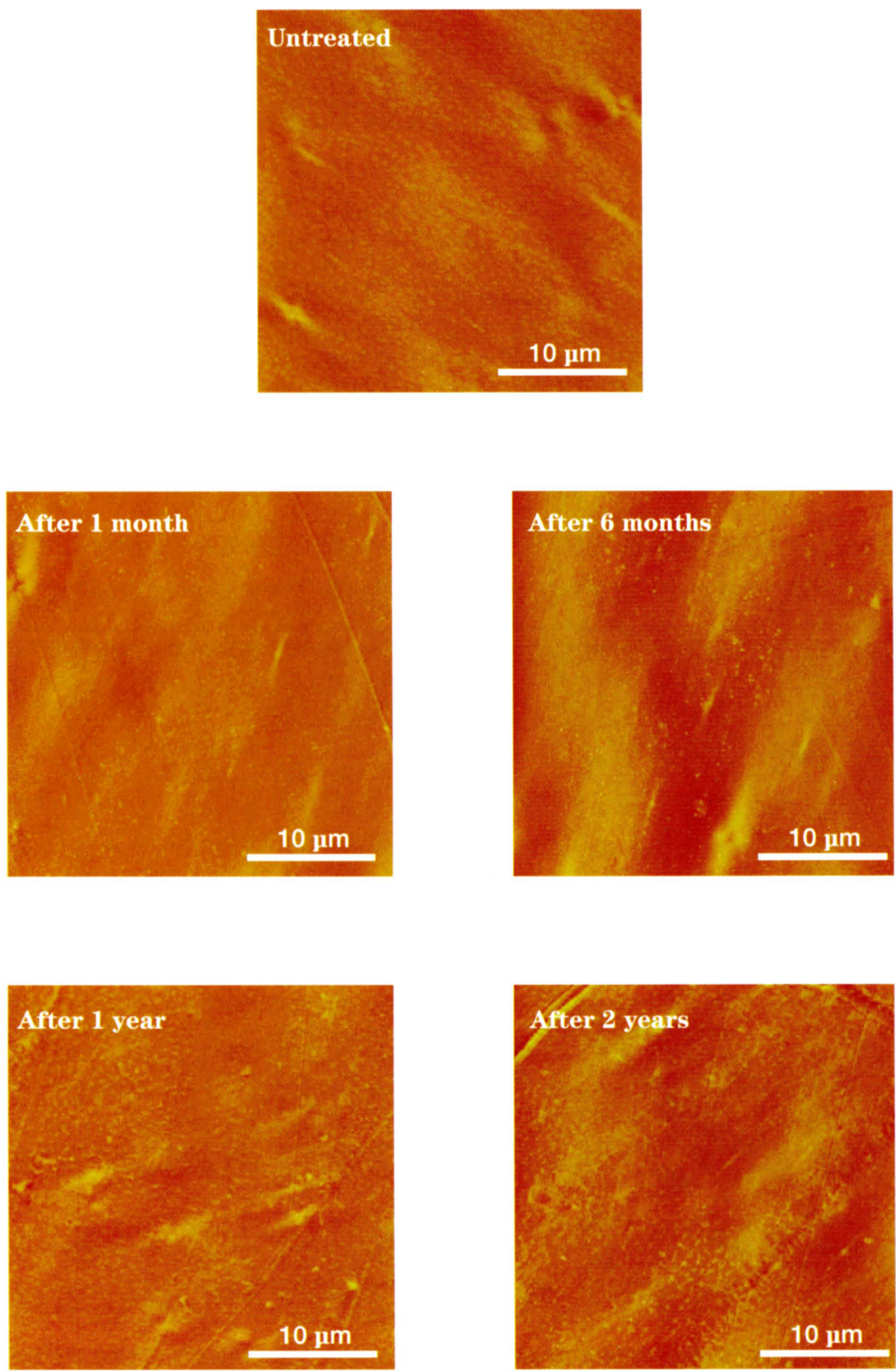

Fig. 5. AFM images of the surfaces of PLA film treated by in-soil burial test. 
$\mathrm{MgCO}_{3}$ or $\mathrm{MgO}$. The $\mathrm{CaCO}_{3}$ is the most popular filler used practically in papermaking. Solubility of these fillers in water is quite low; however a part is dissolved and the $\mathrm{pH}$ value increases over 7 . Figure 6 displays the degradation behavior of PLLA and PDLLA in the filler suspension at $20^{\circ} \mathrm{C}$ for 12 hours or at $80^{\circ} \mathrm{C}$ for 6 hours. The remaining weight (RW) ratio was calculated by dividing the sample weight after treated by the original sample weight. Only $\mathrm{CaO}$ of four fillers made a contribution to the decomposition; and the $\mathrm{CaO}$ played a significant role in enhancing the PLA degradation even at room temperature. There was less than $10 \%$ in the RW ratio; however it may be a sufficient effect on the biodegradation for a long period. Paper-based substrates immediately deteriorate in soil (Kanie et al., 2002), and a part of fillers contained in the paper is dissolved on contact with water in soil. Sequentially, a local alkaline circumstance would promote the initial

Table 2. $\mathrm{pH}$ of $2 \%(\mathrm{wt} / \mathrm{vol})$ suspension containing various alkaline fillers.

\begin{tabular}{lr}
\hline Sample & pH \\
\hline $\mathrm{CaCO}_{3}$ & 9.3 \\
$\mathrm{CaO}$ & 12.3 \\
$\mathrm{MgCO}_{3}$ & 10.1 \\
$\mathrm{MgO}$ & 10.6 \\
\hline
\end{tabular}

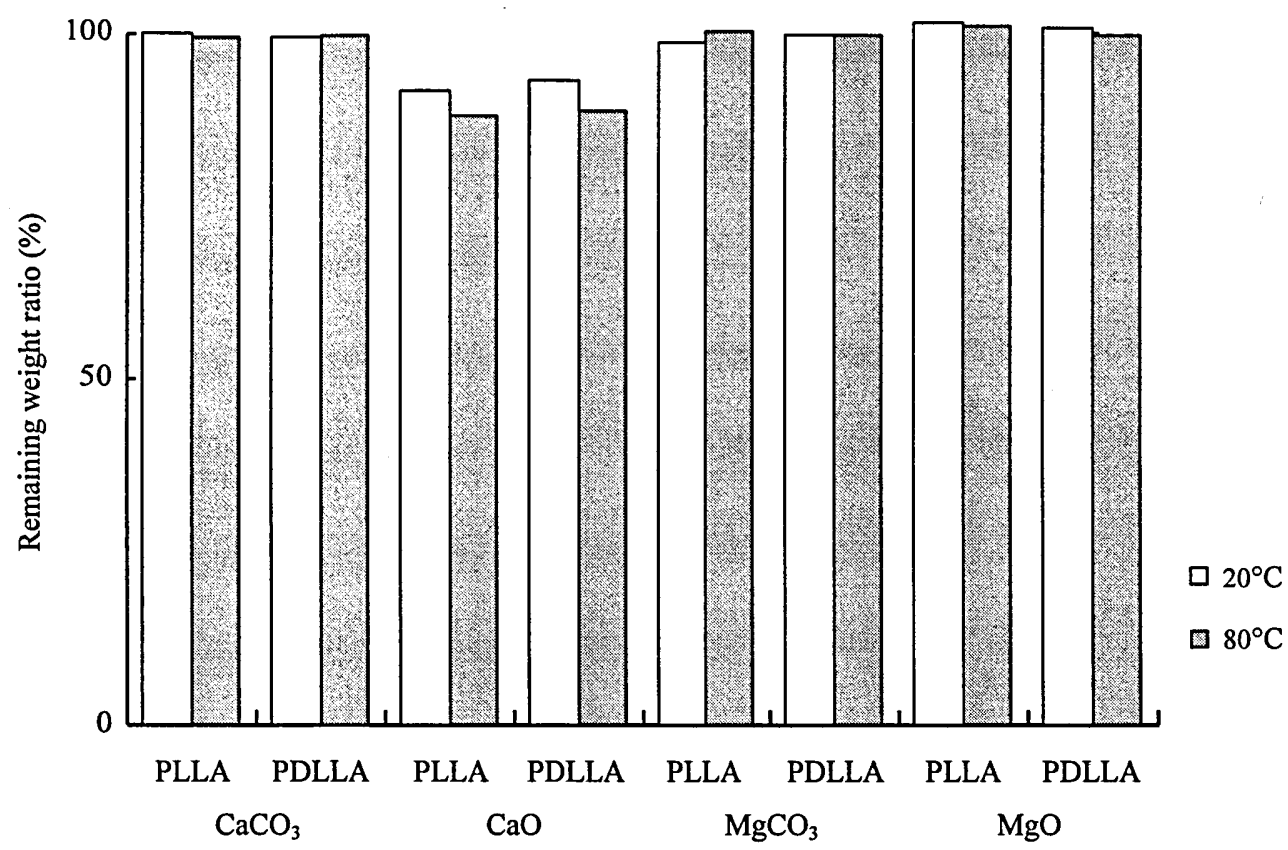

Fig. 6. Weight loss of PLLA and PDLLA treated with the suspension containing alkaline fillers. 
decomposition of the PLA surfaces, resulting in the assistance for biodegradation by in-situ microorganisms. A material design for paper products as a base of PLA composites is multifarious and unrestricted; and thus that conception would be promising for making truly biodegradable composites.

\section{REFERENCES}

Ikada, Y. and H. Tsuji 2000 Biodegradable polyesters for medical and ecological applications. Macromol. Rapid Commun., 21: 117-132

Kanie, O., H. Ishikawa, S. Ohta, T. Kitaoka and H. Tanaka 2002 Study on characteristics of paper laminated with biodegradable plastics: 1. Burial test in soil. J. Fac. Agr., Kyushu Univ., 47: 89-96

Mayumi, A., O. Kanie, T. Kitaoka, H. Wariishi and H. Tanaka 2003 Study on characteristics of paper laminated with biodegradable plastics: 2 . Analytical characterization on chemical and biological degradation. J. Fac. Agr., Kyushu Univ., 48: 85-95

Mohanty, A. K., M. Misra and G. Hinrichsen 2000 Biofibers, biodegradable polymers and biocomposites: An overview. Macromol. Mat. Eng., 276: 1-24

Tsuji, H. and H. Muramatsu 2001 Blends of aliphatic polyesters: V Non-enzymatic and enzymatic hydrolysis of blends from hydrophobic poly (L-lactide) and hydrophilic poly (vinyl alcohol). Polym. Degradation Stability, 71: 403-413

Yamamoto, H., T. Kitaoka and H. Tanaka 2003 Sizing response factors characterized by XPS/PyGC comparative analysis. Sen'i Gakkaishi, 59: 266-271 